\title{
Partitions of Complete Geometric Graphs into Plane Trees ${ }^{\star}$
}

\author{
Prosenjit Bose ${ }^{1}$, Ferran Hurtado ${ }^{2}$, \\ Eduardo Rivera-Campo ${ }^{3}$, and David R. Wood ${ }^{1,4}$ \\ 1 School of Computer Science, Carleton University, \\ Ottawa, Canada \\ $\{$ jit, davidw\}@scs.carleton.ca \\ 2 Departament de Matemàtica Aplicada II, Universitat Politècnica de Catalunya, \\ Barcelona, Spain \\ Ferran.Hurtado@upc.es \\ 3 Departamento de Matemáticas, Universidad Autónoma Metropolitana, \\ Iztapalapa, México \\ erc@xanum.uam.mx \\ 4 Department of Applied Mathematics, Charles University, \\ Prague, Czech Republic
}

\begin{abstract}
Consider the following open problem: does every complete geometric graph $K_{2 n}$ have a partition of its edge set into $n$ plane spanning trees? We approach this problem from three directions. First, we study the case of convex geometric graphs. It is well known that the complete convex graph $K_{2 n}$ has a partition into $n$ plane spanning trees. We characterise all such partitions. Second, we give a sufficient condition, which generalises the convex case, for a complete geometric graph to have a partition into plane spanning trees. Finally, we consider a relaxation of the problem in which the trees of the partition are not necessarily spanning. We prove that every complete geometric graph $K_{n}$ can be partitioned into at most $n-\sqrt{n / 12}$ plane trees.
\end{abstract}

\section{Introduction}

A geometric graph $G$ is a pair $(V(G), E(G))$ where $V(G)$ is a set of points in the plane in general position (that is, no three are collinear), and $E(G)$ is a set of closed segments with endpoints in $V(G)$. Elements of $V(G)$ are vertices and elements of $E(G)$ are edges. An edge with endpoints $v$ and $w$ is denoted by $\{v, w\}$ or $v w$ when convenient. A geometric graph can be thought of as a straight-line drawing of its underlying (abstract) graph. A geometric graph is

\footnotetext{
* Research of all the authors was completed in the Departament de Matemàtica Aplicada II, Universitat Politècnica de Catalunya, Barcelona, Spain. Research of P. Bose supported by NSERC. Research of F. Hurtado supported by projects DURSI 2001SGR00224, MCYT-BFM2001-2340, MCYT-BFM2003-0368 and Gen. Cat 2001SGR00224. Research of E. Rivera-Campo supported by MECD, Spain and Conacyt, México. Research of D. R. Wood supported by NSERC and COMBSTRU.
} 
plane if no two edges cross. A tree is an acyclic connected graph. A subgraph $H$ of a graph $G$ is spanning if $V(H)=V(G)$. We are motivated by the following beautiful question.

Open Problem 1. Does every complete geometric graph with an even number of vertices have a partition of its edge set into plane spanning trees?

Since $K_{n}$, the complete graph on $n$ vertices, has $\frac{1}{2} n(n-1)$ edges and a spanning tree has $n-1$ edges, there are $n / 2$ trees in such a partition, and $n$ is even. We approach this problem from three directions. In Section 2 we study the case of convex geometric graphs. We characterise the partitions of the complete convex graph into plane spanning trees. Section 3 describes a sufficient condition, which generalises the convex case, for a complete geometric graph to have a partition into plane spanning trees. In Section 4 we consider a relaxation of Open Problem 1 in which the trees of the partition are not necessarily spanning.

It is worth mentioning that decompositions of (abstract) graphs into trees have attracted much interest. In particular, Nash-Williams [5] obtained necessary and sufficient conditions for a graph to admit $k$ edge-disjoint spanning trees, and Ringel's Conjecture and the Graceful Tree Conjecture about ways of decomposing complete graphs into trees are among the most outstanding open problems in the field. Nevertheless the non-crossing property that we require in our geometric setting changes the problems drastically.

\section{Convex Graphs}

A convex graph is a geometric graph with the vertices in convex position. An edge on the convex hull of a convex graph is called a boundary edge. Two convex graphs are isomorphic if the underlying graphs are isomorphic and the clockwise ordering of the vertices around the convex hull is preserved under this isomorphism. Suppose that $G_{1}$ and $G_{2}$ are isomorphic convex graphs. Then two edges cross in $G_{1}$ if and only if the corresponding edges in $G_{2}$ also cross. That is, in a convex graph, it is only the order of the vertices around the convex hull that determines edge crossings - the actual coordinates of the vertices are not important.

It is well known that Open Problem 1 has an affirmative solution in the case of convex complete graphs. That is, every convex complete graph $K_{2 n}$ can be partitioned into $n$ plane trees, and since the book thickness of $K_{2 n}$ equals $n$, this bound is optimal even for partitions into plane subgraphs [2]. In this section we characterise the solutions to Open Problem 1 in the convex case. In other words, we characterise the book embeddings of the complete graph in which each page is a spanning tree.

First some well known definitions. A leaf of a tree is a vertex of degree at most one. A leaf-edge of a tree is an edge incident to a leaf. A tree has exactly one leaf if and only if it is a single vertex with no edges. Every tree with at least one edge has at least two leaves. A tree has exactly two leaves if and only if it is a path with at least one edge. Let $T$ be a tree. Let $T^{\prime}$ be the tree obtained by 
deleting the leaves and leaf-edges from $T$. Let $\ell(T)$ be the number of leaves in $T^{\prime}$. A star is a tree with at most one non-leaf vertex. Clearly a tree $T$ is a star if and only if $\ell(T) \leq 1$. A caterpillar is a tree $T$ such that $T^{\prime}$ is a path. The path $T^{\prime}$ is called the spine of the caterpillar. Clearly $T$ is a caterpillar if and only if $\ell(T) \leq 2$. Observe that stars are the caterpillars whose spines consist of a single vertex.

We say a tree $T$ is symmetric if there exists an edge $v w$ of $T$ such that if $A$ and $B$ are the components of $T \backslash v w$ with $v \in A$ and $w \in B$, then there exists a (graph-theoretic) isomorphism between $A$ and $B$ that maps $v$ to $w$.

Theorem 1. Let $T_{1}, T_{2}, \ldots, T_{n}$ be a partition of the edges of the convex complete graph $K_{2 n}$ into plane spanning convex trees. Then $T_{1}, T_{2}, \ldots, T_{n}$ are symmetric convex caterpillars that are pairwise isomorphic. Conversely, for any symmetric convex caterpillar $T$ on $2 n$ vertices, the edges of the convex complete graph $K_{2 n}$ can be partitioned into $n$ plane spanning convex copies of $T$ that are pairwise isomorphic.

We will prove Theorem 1 by a series of lemmas. García et al. [4] proved:

Lemma 1 ([4]). Let $T$ be a tree with at least two edges. In every plane convex drawing of $T$ there are at least $\max \{2, \ell(T)\}$ boundary edges, and there exists a plane convex drawing of $T$ with exactly $\max \{2, \ell(T)\}$ boundary edges, such that if $T$ is not a star then the boundary edges are pairwise non-consecutive.

In what follows $\{0,1, \ldots, 2 n-1\}$ are the vertices of a convex graph in clockwise order around the convex hull. In addition, all vertices are taken modulo $2 n$. That is, vertex $i$ refers to the vertex $j=i \bmod 2 n$. Let $G$ be a convex graph on $\{0,1, \ldots, 2 n-1\}$. For all $0 \leq i, j \leq 2 n-1$, let $G[i, j]$ denote the subgraph of $G$ induced by the vertices $\{i, i+1, \ldots, j\}$.

Lemma 2. For all $n \geq 2$, let $T_{0}, T_{1}, \ldots, T_{n-1}$ be a partition of the convex complete graph $K_{2 n}$ into plane spanning trees. Then (after relabelling the trees) for each $0 \leq i \leq n-1$,

(1) the edge $\{i, n+i\}$ is in $T_{i}$,

(2) $T_{i}$ is a caterpillar with exactly two boundary edges, and

(3) for every non-boundary edge $\{a, b\}$ of $T_{i}$, there is exactly one boundary edge of $T_{i}$ in each of $T_{i}[a, b]$ and $T_{i}[b, a]$.

Proof. The edges $\{\{i, n+i\}: 0 \leq i \leq n-1\}$ are pairwise crossing. Thus each such edge is in a distinct tree. Label the trees such that each edge $\{i, n+i\}$ is in $T_{i}$. Since $n \geq 2$, each $T_{i}$ has at least three edges, and by Lemma 1 , has at least two boundary edges. There are $2 n$ boundary edges in total and $n$ trees. Thus each $T_{i}$ has exactly two boundary edges, and by Lemma $1, \ell\left(T_{i}\right)=2$. For any tree $T, \ell(T) \leq 2$ if and only if $T$ is a caterpillar. Thus each $T_{i}$ is a caterpillar. Let $\{a, b\}$ be a non-boundary edge in some $T_{i}$. Then $T_{i}[a, b]$ has at least one boundary edge of $T_{i}$, as otherwise $T_{i}[a, b]$ would be a convex tree on at least three vertices with only one boundary edge (namely, $\{a, b\}$ ), which contradicts Lemma 1 . Similarly $T_{i}[b, a]$ has at least one boundary edge of $T_{i}$. Thus each of $T_{i}[a, b]$ and $T_{i}[b, a]$ has exactly one boundary edge of $T_{i}$. 
Lemma 3. Let $\{i, j\}$ be a non-boundary edge of a plane convex spanning caterpillar $T$ such that $T[i, j]$ has exactly one boundary edge of $T$. Then exactly one of $\{i, j-1\}$ and $\{j, i+1\}$ is an edge of $T$.

Proof. If both $\{i, j-1\}$ and $\{j, i+1\}$ are in $T$ then they cross, unless $j-1=i+1$ in which case $T$ contains a 3 -cycle. Thus at most one of $\{i, j-1\}$ and $\{j, i+1\}$ is in $T$.

Suppose, for the sake of contradiction, that neither $\{i, j-1\}$ nor $\{j, i+1\}$ are edges of $T$. Since $T$ is spanning, there is an edge $\{i, a\}$ or $\{j, a\}$ in $T$ for some vertex $i+1<a<j-1$. Without loss of generality $\{i, a\}$ is this edge, as illustrated in Figure 1.

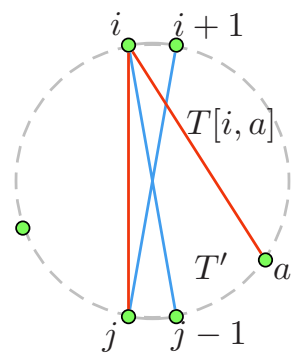

Fig. 1. One of $\{i, j-1\}$ and $\{j, i+1\}$ is an edge of $T$.

Since $i, i+1$ and $a$ are distinct vertices of $T[i, a]$, the subtree $T[i, a]$ has at least three vertices, and by Lemma 1 , has at least two boundary edges, one of which is $\{i, a\}$. Thus $T[i, a]$ has at least one boundary edge that is also a boundary edge of $T$. Now consider the subtree $T^{\prime}$ of $T$ induced by $\{i\} \cup\{a, a+1, \ldots, j\}$. Then $i, a, j-1$ and $j$ are distinct vertices of $T^{\prime}$, and $T^{\prime}$ has at least four vertices. Since $\{i, j-1\}$ is not an edge of $T$, and thus not an edge of $T^{\prime}$, the subtree $T^{\prime}$ is not a star. By Lemma $1, T^{\prime}$ has at least two non-consecutive boundary edges, at most one of which is $\{i, j\}$ or $\{i, a\}$. Thus $T^{\prime}$ has at least one boundary edge that is also a boundary edge of $T$.

No boundary edge of $T$ can be in both $T[i, a]$ and $T^{\prime}$. Thus we have shown that $T[i, j]$ has at least two boundary edges of $T$, which is the desired contradiction.

In what follows we say an edge $e=\{i, j\}$ has span

$$
\operatorname{span}(e)=\min \{(i-j) \bmod 2 n,(j-i) \bmod 2 n\} .
$$

That is, $\operatorname{span}(e)$ is the number of edges in a shortest path between $i$ and $j$ that is contained in the convex hull.

Lemma 4. Let $\{i, j\}$ be an edge of a plane convex spanning caterpillar $T$ such that $1 \leq j-i \leq n$, and $T[i, j]$ has exactly one boundary edge of $T$. Then $T[i, j]$ has exactly one edge of span $k$ for each $1 \leq k \leq j-i$. Moreover for each such $k \geq 2$ the edge of span $k$ has an endpoint in common with the edge of span $k-1$, and the other two endpoints are consecutive on the convex hull. 
Proof. If $j-i=1$ then $\{i, j\}$ is a boundary edge, and the result is trivial. Otherwise $\{i, j\}$ is not a boundary edge. By Lemma 3, exactly one of the edges $\{i, j-1\}$ and $\{j, i+1\}$ is in $T$. Without loss of generality $\{i, j-1\}$ is in $T$. Thus the edge of span $j-i$ has an endpoint in common with the edge of span $j-i-1$, and the other two endpoints are consecutive on the convex hull. The result follows by induction (on span) applied to the edge $\{i, j-1\}$.

Theorem 2 below is the main theorem of this section, and its proof is illustrated in Figure 4. Let $e=\{a, b\}$ be an edge in the convex complete graph $K_{2 n}$. Then $e+i$ denotes the edge $\{a+i, b+i\}$. For a set $X$ of edges, $X+i=\{e+i$ : $e \in X\}$, and $X^{(k)}=\{e \in X, \operatorname{span}(e) \geq k\}$.

Theorem 2. Let $T_{0}, T_{1}, \ldots, T_{n-1}$ be a partition of the edges of the convex complete graph $K_{2 n}$ into plane spanning convex trees. Then $T_{0}, T_{1}, \ldots, T_{n-1}$ are pairwise isomorphic symmetric convex caterpillars.

Proof. By Lemma 2, for each $0 \leq i \leq n-1, T_{i}$ is a caterpillar with two boundary edges, the edge $\{i, n+i\}$ is in $T_{i}$, and for every non-boundary edge $\{a, b\}$ of $T_{i}$, there is exactly one boundary edge of $T_{i}$ in each of $T_{i}[a, b]$ and $T_{i}[b, a]$.

Let $H=T_{0}[0, n]$. Since $\{0, n\}$ is an edge of $H$, by Lemma $4, H$ has exactly one edge of span $k$ for each $1 \leq k \leq n$. Furthermore, for each $1 \leq k \leq n-1$, the edge of span $k$ has an endpoint in common with the edge of span $k+1$, and the other two endpoints are consecutive on the convex hull. Let $h_{k}=\left\{x_{k}, x_{k}+k\right\}$ denote the edge of span $k$ in $H$. For each $1 \leq k \leq n-1$, if $h_{k} \cap h_{k+1}=x_{k}+k$ $\left(=x_{k+1}+k+1\right)$ then we say the $k$-direction is 'clockwise'. Otherwise, $h_{k} \cap$ $h_{k+1}=x_{k}\left(=x_{k+1}\right)$, and we say the $k$-direction is 'anticlockwise', as illustrated in Figure 2.

We will prove that $H$ determines the structure of all the trees $T_{0}, T_{1}, \ldots, T_{n-1}$. We proceed by downwards induction on $k=n, n-1, \ldots, 1$ with the hypothesis that for all $0 \leq i \leq n-1$,

$$
T_{i}^{(k)}=\left(H^{(k)}+i\right) \cup\left(H^{(k)}+n+i\right)
$$

Consider the base case with $k=n$. The only edge in $H$ of span $n$ is $\{0, n\}$. Thus $H^{(n)}=\{0, n\}$, which implies that $H^{(n)}+i=\{i, n+i\}$, and $H^{(n)}+n+i=$

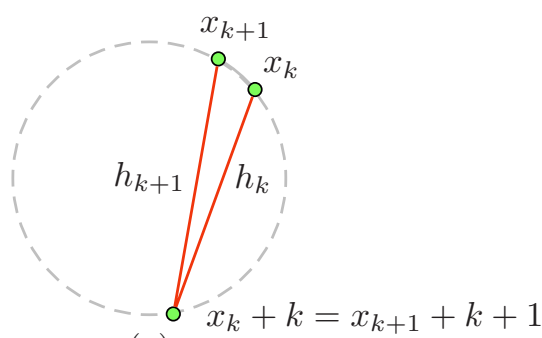

(a)

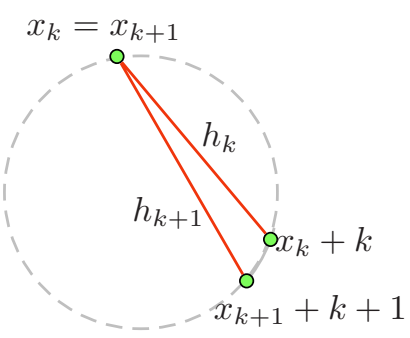

(b)

Fig. 2. $k$-direction is (a) clockwise and (b) anticlockwise. 
$\{n+i, 2 n+i\}=\{i, n+i\}$. Thus the right-hand side of (1) is $\{i, n+i\}$. The only edge in $T_{i}$ of $\operatorname{span} n$ is $\{i, n+i\}$. Thus $T_{i}^{(n)}=\{i, n+i\}$, and (1) is satisfied for $k=n$.

Now suppose that (1) holds for some $k+1 \geq 2$. We will prove that (1) holds for $k$. Suppose that the $k$-direction is clockwise. (The case in which the $k$-direction as anticlockwise is symmetric.) We proceed by induction on $j=$ $0,1, \ldots, 2 n-1$ with the hypothesis:

$$
\text { the edge }\left\{x_{k}+j, x_{k}+k+j\right\} \text { is in the tree } T_{j} \bmod n .
$$

The base case with $j=0$ is immediate since by definition, $\left\{x_{k}, x_{k}+k\right\} \in$ $E\left(T_{0}\right)$. Suppose that $\left\{x_{k}+j, x_{k}+k+j\right\} \in E\left(T_{j \bmod n}\right)$ for some $0 \leq j<2 n-1$. Consider the edge $e=\left\{x_{k}+j, x_{k}+k+j+1\right\}$. Since the $k$-direction is clockwise, $x_{k}=x_{k+1}+1$ and $x_{k}+k=x_{k+1}+k+1$. Thus $e=\left\{x_{k+1}+1+j, x_{k+1}+k+1+\right.$ $j+1\}=\left\{x_{k+1}, x_{k+1}+k+1\right\}+j+1=h_{k}+j+1$. Hence $e \in H+j+1$, and since $e$ has span $k+1, e \in H^{(k+1)}+j+1$. By induction from (1), $e \in T_{(j+1) \bmod n}^{(k+1)}$, as illustrated in Figure 3.

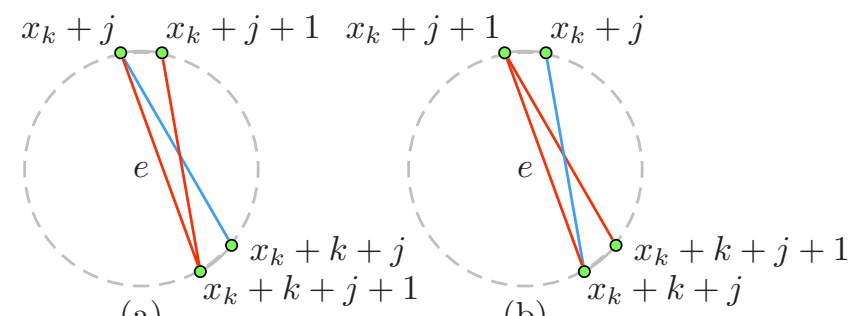

(a)

(b)

Fig. 3. $k$-direction is (a) clockwise and (b) anticlockwise.

By Lemma 3 applied to $e$, which is a non-boundary edge of $T_{(j+1)} \bmod n$, exactly one of $\left\{x_{k}+j, x_{k}+k+j\right\}$ and $\left\{x_{k}+j+1, x_{k}+k+j+1\right\}$ is an edge of $T_{(j+1) \bmod n}$. By induction from (2), $\left\{x_{k}+j, x_{k}+k+j\right\} \in T_{j \bmod n}$. Thus $\left\{x_{k}+j+1, x_{k}+k+j+1\right\} \in T_{(j+1)} \bmod n$. That is, (2) holds for $j+1$. Therefore for all $0 \leq j \leq 2 n-1$, the edge $\left\{x_{k}+j, x_{k}+k+j\right\}$ is in $T_{j \bmod n}$. That is, $h_{k}+j$ is in $T_{j \bmod n}$. By (1) for $k+1$ we have that (1) holds for $k$.

By (1) with $k=1$, each tree $T_{i}$ can be expressed as $T_{i}=(H+i) \cup(H+n+i)$. Clearly $H \cup(H+n)$ is a symmetric convex caterpillar. Thus each $T_{i}$ is a translated copy of the same symmetric convex caterpillar. Therefore $T_{0}, T_{1}, \ldots, T_{n-1}$ are pairwise isomorphic symmetric convex caterpillars.

Theorem 3. For any symmetric convex caterpillar $T$ on $2 n$ vertices, the edges of the convex complete graph $K_{2 n}$ can be partitioned into $n$ plane spanning pairwise isomorphic convex copies of $T$.

Proof. Say $V\left(K_{2 n}\right)=\{0,1, \ldots, 2 n-1\}$ in clockwise order around the convex hull. Let $\{0, n\}$ be the edge of $T$ such that after deleting $\{0, n\}, A$ and $B$ are 

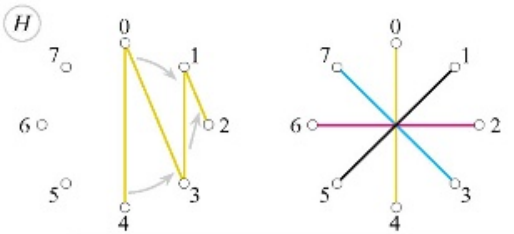

$$
\begin{array}{ll}
h_{4}=\{0,4\} & \\
h_{3}=\{0,3\} & \text { 3-direction is anticlockwise } \\
h_{2}=\{1,3\} & \text { 2-direction is clockwise } \\
h_{1}=\{1,2\} & \text { 1-direction is anticlockwise }
\end{array}
$$
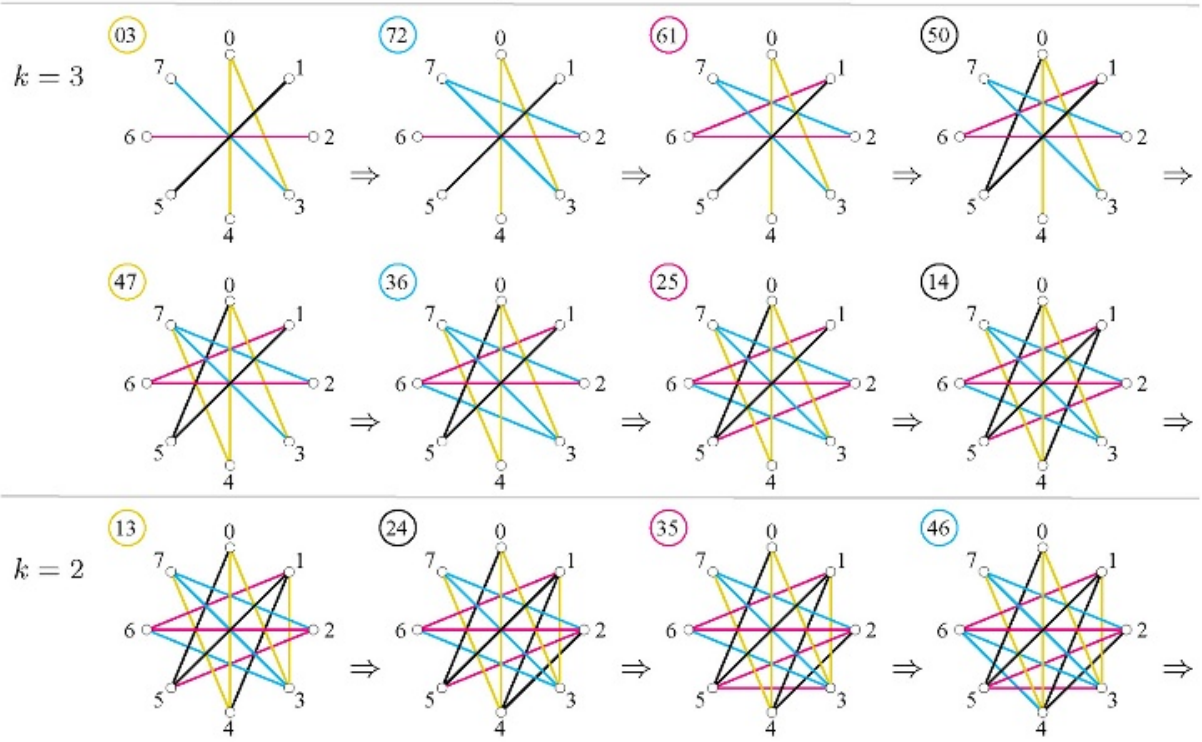

(24)

(35)
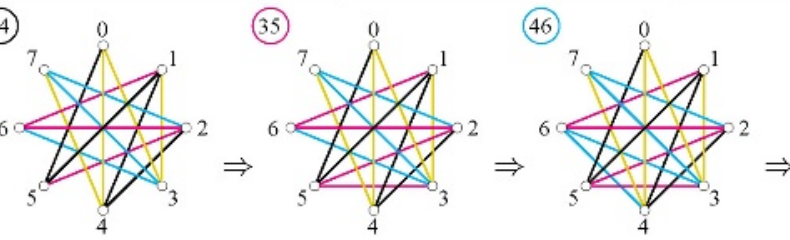

57
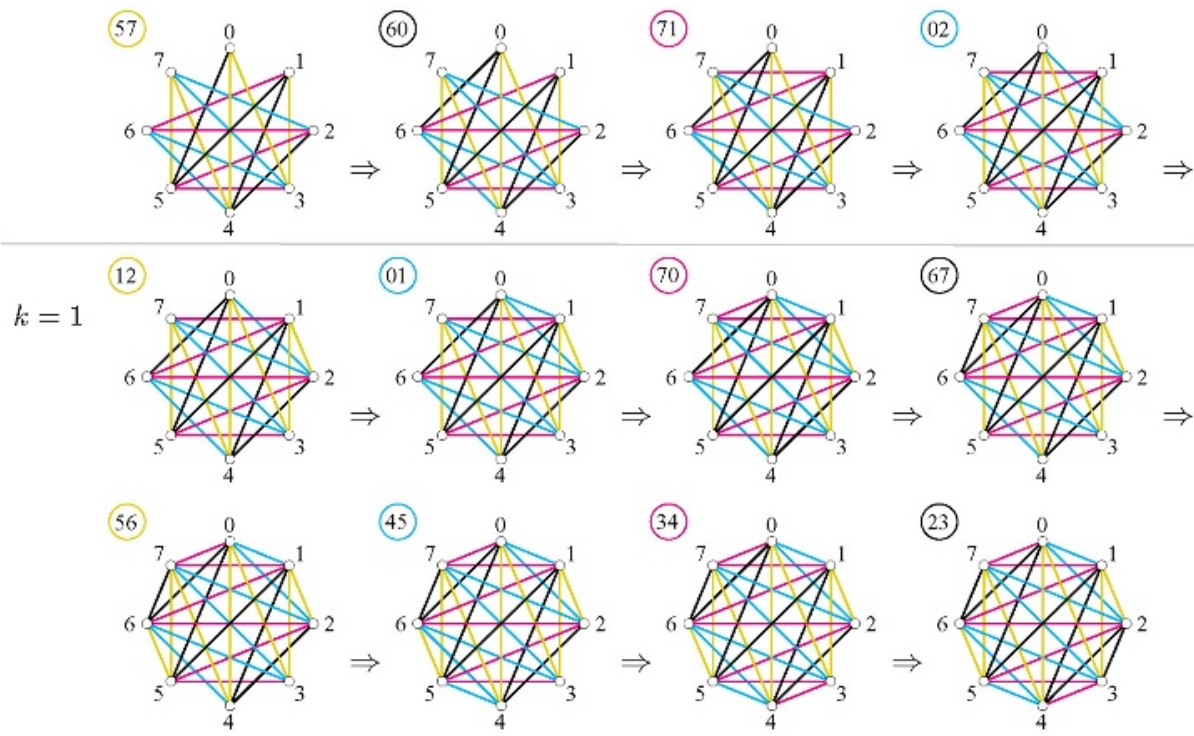

(45)

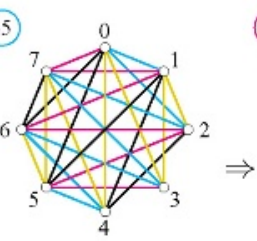

(34)

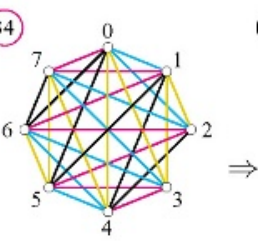

(23)

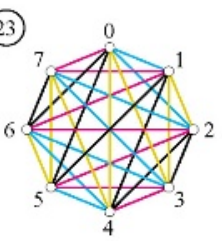

Fig. 4. Illustration for Theorem 2 with $n=4$. 
the components with $0 \in A$ and $n \in B$, and there exists a (graph-theoretic) isomorphism between $A$ and $B$ that maps 0 to $n$. It is easily seen that $A$ has a plane representation on the vertices $\{0,1, \ldots, n\}$. For each $0 \leq i \leq n-1$, let $T_{i}=(A+i) \cup(A+n+i)$. Then as in Theorem $2, T_{0}, T_{1}, \ldots, T_{n-1}$ is partition of $K_{2 n}$ into plane spanning pairwise isomorphic convex copies of $T$.

Observe that Theorems 2 and 3 together prove Theorem 1.

\section{A Sufficient Condition}

In this section we prove the following sufficient condition for a complete geometric graph to have an affirmative solution to Open Problem 1. A double star is a tree with at most two non-leaf vertices.

Theorem 4. Let $G$ be a complete geometric graph $K_{2 n}$. Suppose that there is a set $\mathcal{L}$ of pairwise non-parallel lines with exactly one vertex of $G$ in each open unbounded region formed by $\mathcal{L}$. Then $E(G)$ can be partitioned into plane spanning double stars (that are pairwise graph-theoretically isomorphic).

Observe that in a double star, if there are two non-leaf vertices $v$ and $w$ then they must be adjacent, in which case we say $v w$ is the root edge.

Lemma 5. Let $P$ be a set of points in general position. Let $L$ be a line with $L \cap P=\emptyset$. Let $H_{1}$ and $H_{2}$ be the half-planes defined by L. Let $v$ and $w$ be points such that $v \in P \cap H_{1}$ and $w \in P \cap H_{2}$. Let $T(P, L, v, w)$ be the geometric graph with vertex set $P$ and edge set

$$
\{v w\} \cup\left\{v x: x \in(P \backslash\{v\}) \cap H_{1}\right\} \cup\left\{w y: y \in(P \backslash\{w\}) \cap H_{2}\right\} .
$$

Then $T(P, L, v, w)$ is a plane double star with root edge vw.

Proof. The set of edges incident to $v$ form a star. Regardless of the point set, a geometric star is always plane. Thus no two edges incident to $v$ cross. Similarly no two edges incident to $w$ cross. No edge incident to $v$ crosses an edge incident to $w$ since such edges are separated by $L$, as illustrated in Figure 5.

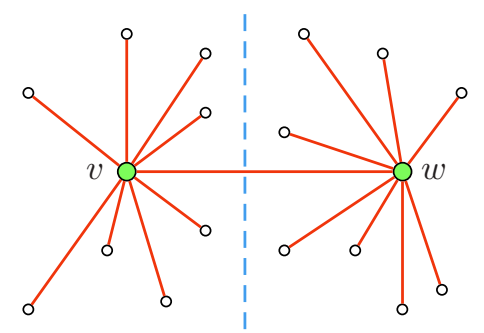

Fig. 5. A plane double star separated by a line. 
Lemma 6. Let $P$ be a set of points in general position. Let $L_{1}$ and $L_{2}$ be nonparallel lines with $L_{1} \cap P=L_{2} \cap P=\emptyset$. Let $v, w, x, y$ be points in $P$ such that $v, w, x, y$ are in distinct quarter-planes formed by $L_{1}$ and $L_{2}$, with each pair $(v, w)$ and $(x, y)$ in opposite quarter-planes. (Note that this does not imply that vw and $x y$ cross.) Let $T_{1}$ and $T_{2}$ be the plane double stars $T_{1}=T\left(P, L_{1}, v, w\right)$ and $T_{2}=T\left(P, L_{2}, x, y\right)$. Then $E\left(T_{1}\right) \cap E\left(T_{2}\right)=\emptyset$.

Proof. Suppose, for the sake of contradiction, that there is an edge $e \in E\left(T_{1}\right) \cap$ $E\left(T_{2}\right)$. All edges of $T_{1}$ are incident to $v$ or $w$, and all edges of $T_{2}$ are incident to $x$ or $y$. Thus $e \in\{v x, v w, v y, x w, x y, w y\}$. By assumption, $v, w, x, y$ are in distinct quarter-planes formed by $L_{1}$ and $L_{2}$, with each pair $(v, w)$ and $(x, y)$ in opposite quarter-planes. Thus $e$ crosses at least one of $L_{1}$ and $L_{2}$. Without loss of generality $e$ crosses $L_{1}$. Since $e \in E\left(T_{1}\right)$, and the only edge of $T_{1}$ that crosses $L_{1}$ is the root edge $v w$, we have $e=v w$. Since all edges of $T_{2}$ are incident to $x$ or $y$ and $v, w, x, y$ are distinct, we have $e \notin E\left(T_{2}\right)$, which is the desired contradiction. Therefore $E\left(T_{1}\right) \cap E\left(T_{2}\right)=\emptyset$, as illustrated in Figure 6 .

Proof (of Theorem 4). As illustrated in Figure 7, let $C$ be a circle such that the vertices of $G$ and the intersection point of any two lines in $\mathcal{L}$ are in the interior of $C$. The intersection points of $C$ and the lines in $\mathcal{L}$ partition $C$ into $2 n$ consecutive components $C_{0}, C_{1}, \ldots, C_{2 n-1}$, each corresponding to a region containing a single vertex of $G$. Let $i$ be the vertex in the region corresponding to $C_{i}$. Label the lines $L_{0}, L_{1}, \ldots, L_{n-1}$ so that for each $0 \leq i \leq n-1$, the components $C_{i}$ and $C_{i+n}$ run from $C \cap L_{i}$ to $C \cap L_{(i+1) \bmod n}$ in the clockwise direction.

For each $0 \leq i \leq n-1$, let $T_{i}$ be the double star $T\left(V(G), L_{i}, i, i+n\right)$. By Lemma 5 , each $T_{i}$ is plane. Since $V\left(T_{i}\right)=V(G), T_{i}$ is a spanning tree of $G$. For all $0 \leq i<j \leq n-1$, the points $i, i+n, j, j+n$ are in distinct quarter-planes

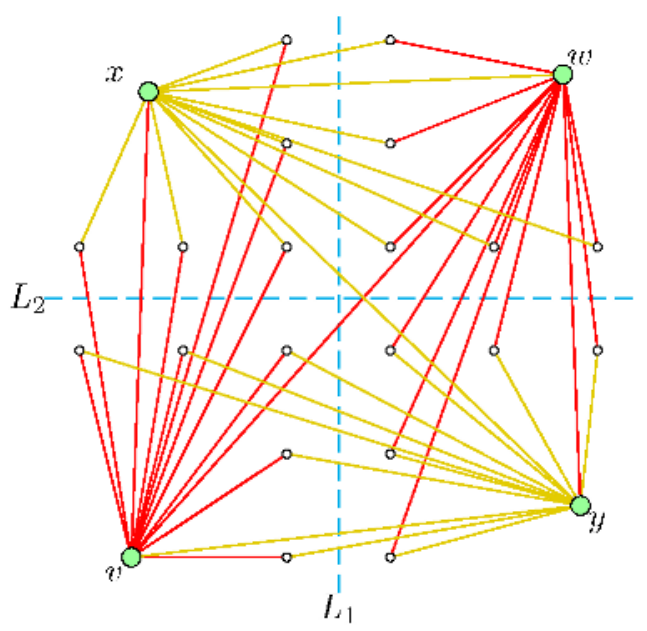

Fig. 6. Plane spanning double stars are edge-disjoint. 


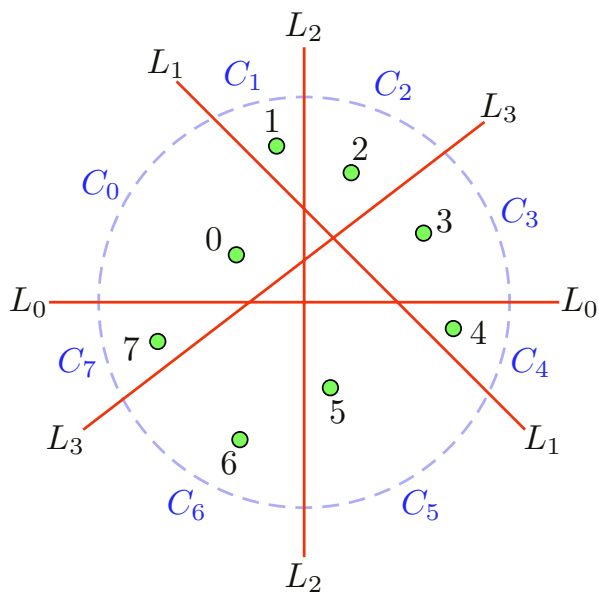

Fig. 7. Example of Theorem 4 with $n=4$.

formed by $L_{i}$ and $L_{j}$, with each pair $(i, i+n)$ and $(j, j+n)$ in opposite quarterplanes. Thus, by Lemma $6, E\left(T_{i}\right) \cap E\left(T_{j}\right)=\emptyset$. Since each $T_{i}$ has $2 n-1$ edges, and there are $n(2 n-1)$ edges in total, $T_{0}, T_{1}, \ldots, T_{n-1}$ is the desired partition of $E(G)$.

Note that each line in $\mathcal{L}$ in Theorem 4 is a halving line. Pach and Solymosi [6] proved a related result: a complete geometric graph on $2 n$ vertices has $n$ pairwise crossing edges if and only if it has precisely $n$ halving lines.

\section{Relaxations}

We now drop the requirement that our plane trees be spanning. Thus we need not restrict ourselves to complete graphs with an even number of vertices. Theorem 4 generalises as follows.

Theorem 5. Let $G$ be a complete geometric graph $K_{n}$. Suppose that there is a set $\mathcal{L}$ of pairwise non-parallel lines with at least one vertex of $G$ in each open unbounded region formed by $\mathcal{L}$. Then $E(G)$ can be partitioned into $n-|\mathcal{L}|$ plane trees.

Proof. Let $P$ be a set consisting of exactly one vertex in each open unbounded region formed by $\mathcal{L}$. Then $|P|=2|\mathcal{L}|$. By Theorem 4 , the induced subgraph $G[P]$ can be partitioned into $\frac{1}{2}|P|$ plane double stars. The edges incident to a vertex not in $P$ can be covered by $n-|P|$ spanning stars, one rooted at each of the vertices not in $P$. Clearly a star is plane regardless of the vertex positions. Edges with both endpoints not in $P$ can be placed in the star rooted at either endpoint. In total we have $\frac{1}{2}|P|+(n-|P|)=n-\frac{1}{2}|P|=n-|\mathcal{L}|$ plane trees.

Lemma 7. Every complete geometric graph $K_{n}$ with $k$ pairwise crossing edges can be partitioned into $n-k$ plane trees. 
Proof. Let $E=\left\{e_{i}: 1 \leq i \leq k\right\}$ be a set of $k$ pairwise crossing edges. For each $1 \leq i \leq k$, let $L_{i}$ be the line obtained by extending the segment $e_{i}$, and rotating it about the midpoint of $e_{i}$ by some angle of $\epsilon$ degrees. Clearly there exists an $\epsilon$ such that each edge $e_{i}$ crosses every line $L_{j}$. Thus there is one endpoint of an edge in $E$ in each open unbounded region formed by $L_{1}, L_{2}, \ldots, L_{k}$. The result follows from Theorem 5 .

Aronov et al. [1] proved that every complete geometric graph $K_{n}$ has at least $\sqrt{n / 12}$ pairwise crossing edges. Thus Lemma 7 implies:

Corollary 1. Every complete geometric graph $K_{n}$ can be partitioned into at most $n-\sqrt{n / 12}$ plane trees.

We conclude with a seemingly easier problem than Open Problem 1.

Open Problem 2. Can the edges of every complete geometric graph $K_{n}$ be partitioned into at most $n / c$ plane subgraphs, for some constant $c>1$ ?

Of course $c<2$ since $n / 2$ edges may be pairwise crossing. Dillencourt et al. [3] defined the geometric thickness of an (abstract) graph $G$ to be the minimum $k$ such that $G$ has a representation as a geometric graph whose edges can be partitioned into $k$ plane subgraphs. They proved that the geometric thickness of $K_{n}$ is between $\lceil(n / 5.646)+0.342\rceil$ and $\lceil n / 4\rceil$.

\section{References}

1. Boris Aronov, Paul Erdős, Wayne Goddard, Daniel J. Kleitman, Michael Klugerman, János Pach, and Leonard J. Schulman. Crossing families. Combinatorica, 14(2):127-134, 1994.

2. Frank R. Bernhart and Paul C. Kainen. The book thickness of a graph. $J$. Combin. Theory Ser. B, 27(3):320-331, 1979.

3. Michael B. Dillencourt, David Eppstein, and Daniel S. Hirschberg. Geometric thickness of complete graphs. J. Graph Algorithms Appl., 4(3):5-17, 2000.

4. Alfredo García, Carmen Hernando, Ferran Hurtado, Marc Noy, and JAVIER TEJEL. Packing trees into planar graphs. J. Graph Theory, 40(3):172-181, 2002.

5. C. St. J. A. Nash-Williams. Decomposition of finite graphs into forests. J. London Math. Soc., 39:12, 1964.

6. János PACH And József Solymosi. Halving lines and perfect cross-matchings. In Advances in discrete and computational geometry, volume 223 of Contemp. Math., pages 245-249. Amer. Math. Soc., 1999. 